Washington University School of Medicine Digital Commons@Becker

Open Access Publications

2017

\title{
Is there an association between bone mineral density and mammographic density? A systematic review
}

Jong Min Lee

Washington University School of Medicine in St. Louis

Susan Holley

Washington University School of Medicine in St. Louis

Catherine Appleton

Washington University School of Medicine in St. Louis

Adetunji T. Toriola

Washington University School of Medicine in St. Louis

Follow this and additional works at: https://digitalcommons.wustl.edu/open_access_pubs

Please let us know how this document benefits you.

\section{Recommended Citation}

Lee, Jong Min; Holley, Susan; Appleton, Catherine; and Toriola, Adetunji T., "Is there an association between bone mineral density and mammographic density? A systematic review." Journal of Women's Health. 26, 4. 389-395. (2017).

https://digitalcommons.wustl.edu/open_access_pubs/5795

This Open Access Publication is brought to you for free and open access by Digital Commons@Becker. It has been accepted for inclusion in Open Access Publications by an authorized administrator of Digital Commons@Becker. For more information, please contact vanam@wustl.edu. 


\title{
Is There an Association Between Bone Mineral Density and Mammographic Density? A Systematic Review
}

\author{
Jong Min Lee, MPH, Susan Holley, MD, ${ }^{2}$ Catherine Appleton, $\mathrm{MD}^{2}$, and Adetunji T. Toriola, MD, MPH, $\mathrm{PhD}^{1}$
}

\begin{abstract}
Introduction: Both bone mineral density (BMD) and breast density are related to reproductive hormone levels. This suggests that BMD and breast density could be meaningfully associated, and serve as surrogate markers for breast cancer risk. However, few studies have investigated the association of BMD with percent mammographic density, making it difficult to draw meaningful conclusions.

Materials and Methods: We conducted a systematic review of studies published in electronic databases till April 2016 using the following search terms: "bone density," "bone mineral density," "mammographic breast density," "breast density," and "mammographic density." We identified 203 articles, of which 8 met the inclusion criteria for this review.

Results: BMD does not appear to be associated with percent mammographic density. BMD at the spine was weakly positively associated with percent mammographic density among postmenopausal women who were not hormone users, while BMD at the hip and legs was positively associated with percent mammographic density among premenopausal women. On the other hand, one study reported an inverse association of BMD at the spine and hip with percent mammographic density among perimenopausal women.
\end{abstract}

Conclusion: In this review, we found no evidence of an association between BMD and percent mammographic density.

Keywords: bone mineral density (BMD), mammographic density, menopause

\section{Introduction}

$\mathbf{M}$ AMMOGRAPHIC DENSITY IS a strong risk factor for breast cancer. An increased breast density is associated with a four- to sixfold increased risk of breast cancer. ${ }^{1-4}$ Likewise, some studies have suggested that increased bone mineral density (BMD) might be associated with an increased risk of breast cancer, especially among postmenopausal women. ${ }^{5,6}$ Although the underlying mechanisms are still poorly understood, hormonal exposure is thought to play an important role in driving the association of mammographic density with breast cancer risk. ${ }^{7-10}$ In the Women's Health Initiative (WHI) trial, 1 year of estrogen plus progestin use was associated with a $6 \%$ increase in mammographic density. ${ }^{11}$ Hormonal exposure also plays an important role in BMD. ${ }^{12,13}$ Longer duration of exposure to estrogen is positively associated with BMD. ${ }^{14-16}$ Estrogen deficiency during menopause induces cortical bone loss by inhibiting bone resorption activity, resulting in decreased bone mass. ${ }^{17-19}$ Hence, it is possible that similar etiological pathways involving reproductive hormone exposure may drive the association of mammographic density and BMD with breast cancer risk. On the other hand, adiposity, which is a risk factor for postmenopausal breast cancer (but inversely associated with premenopausal breast cancer), has divergent effects on mammographic density and BMD. ${ }^{20,21}$ While body mass index (BMI) is positively associated with $\mathrm{BMD},{ }^{22,23}$ it is inversely associated with mammographic density. ${ }^{24,25}$ Therefore, the association of BMD with percent mammographic density may be more complex.

Understanding the association of BMD with percent mammographic density might provide better knowledge of how breast cancer intermediaries increase breast cancer risk. Furthermore, if mammographic density is associated with BMD, knowing a woman's breast density might have some utility in predicting $\mathrm{BMD}$, and possibly future fracture risk since $\mathrm{BMD}$ is a strong predictor of future fracture risk. ${ }^{26}$ The

\footnotetext{
${ }^{1}$ Division of Public Health Sciences, Department of Surgery, Siteman Cancer Center, Washington University School of Medicine, St Louis, Missouri.

${ }^{2}$ Division of Diagnostic Radiology, Department of Radiology, Siteman Cancer Center, Washington University School of Medicine, St Louis, Missouri.
} 
few studies that have investigated the association of BMD with percent mammographic density, ${ }^{27-34}$ have produced conflicting results, and no review of the topic has been undertaken. To extend knowledge on the association of BMD with percent mammographic density, we conducted a systematic review of published studies.

\section{Materials and Methods}

We identified studies published between January 2000 and April 2016 using the following databases: PubMed, OVidSP, BioMed Central, EMBASE, Web of Science, and Scopus. Search terms included the following: "bone density," "bone mineral density," "mammographic breast density," "breast density," and "mammographic density." Search queries in the databases yielded 211 articles (Figure 1). Abstracts and results were examined if the articles were relevant to our topic of study. Reference lists of relevant articles were also examined.

From 211 articles, we included studies if they met the following criteria: reported on (1) the association of BMD with percent mammographic density. Hence, studies using Breast Imaging-Reporting and Data System (BI-RADS) classification were not included. The exposure was defined as BMD, while the outcome was defined as percent mammographic density. (2) Publications were in English language. Eight studies met the inclusion criteria. From these eight articles, we extracted information on study design, age, menopausal status, and number of participants, BMD sites, and confounders. Studies were available on the following BMD sites in relation to mammographic density: spine $(N=7)$, hip $(N=6)$, and other sites, including arms, ribs, and overall body $(N=3)$. We extracted data on beta coefficient values, $95 \%$ confidence intervals (CIs), and $p$-values from the original studies.

\section{Results}

\section{$B M D$ at the spine}

Seven studies have investigated the association of BMD at the spine with percent mammographic density ${ }^{27-33}$ (Table 1).
No associations were reported in studies that did not stratify by menopausal status. BMD of the spine was associated with percent mammographic density in two studies of the five studies that stratified by menopausal status. Crandall et al. reported a very weak positive association between BMD at the spine $(\beta=0.099, p=0.08)$ and percent mammographic density among postmenopausal women who were not recent hormone users after adjusting for age, BMI, and cigarette smoking. ${ }^{29}$ In another study, the same authors observed an inverse association between BMD at the spine $(\beta=-20.6$ [95\% CI: -37.2 to -3.9 ],$p<0.05)$ and mammographic density among early perimenopausal women. ${ }^{30}$

\section{$B M D$ at the hip}

Six studies have investigated the association of BMD at the hip with percent mammographic density ${ }^{27,29-33}$ (Table 2). The studies that investigated associations among all women regardless of menopausal status reported no association. ${ }^{27,31,33}$ Five studies stratified their analyses by menopausal status. BMD at the hip was positively associated with mammographic density in two studies: one among premenopausal women $(\beta=0.59, p<0.05)^{33}$ and one among postmenopausal women who were not recent hormone users $(\beta=0.156, p<0.05){ }^{29}$ Conversely, an inverse association was observed among early perimenopausal women $(\beta=-18.8$ [95\% CI: -37.0 to -0.6 ], $p<0.05){ }^{30}$

\section{BMD at other sites (legs, ribs, arms, and whole body)}

Only three studies have investigated the association of BMD at other sites, including ribs, arms, leg, and whole body, with mammographic density ${ }^{28,33,34}$ and two studies reported no associations overall. However, Sung et al. observed a positive association of BMD at the legs with mammographic density among premenopausal women $(\beta=0.59, p<0.05)^{33}$ (Table 3 ). A recent study observed a positive association of BMD at the forearm with percent mammographic density
FIG. 1. Flow diagram of the literature search.

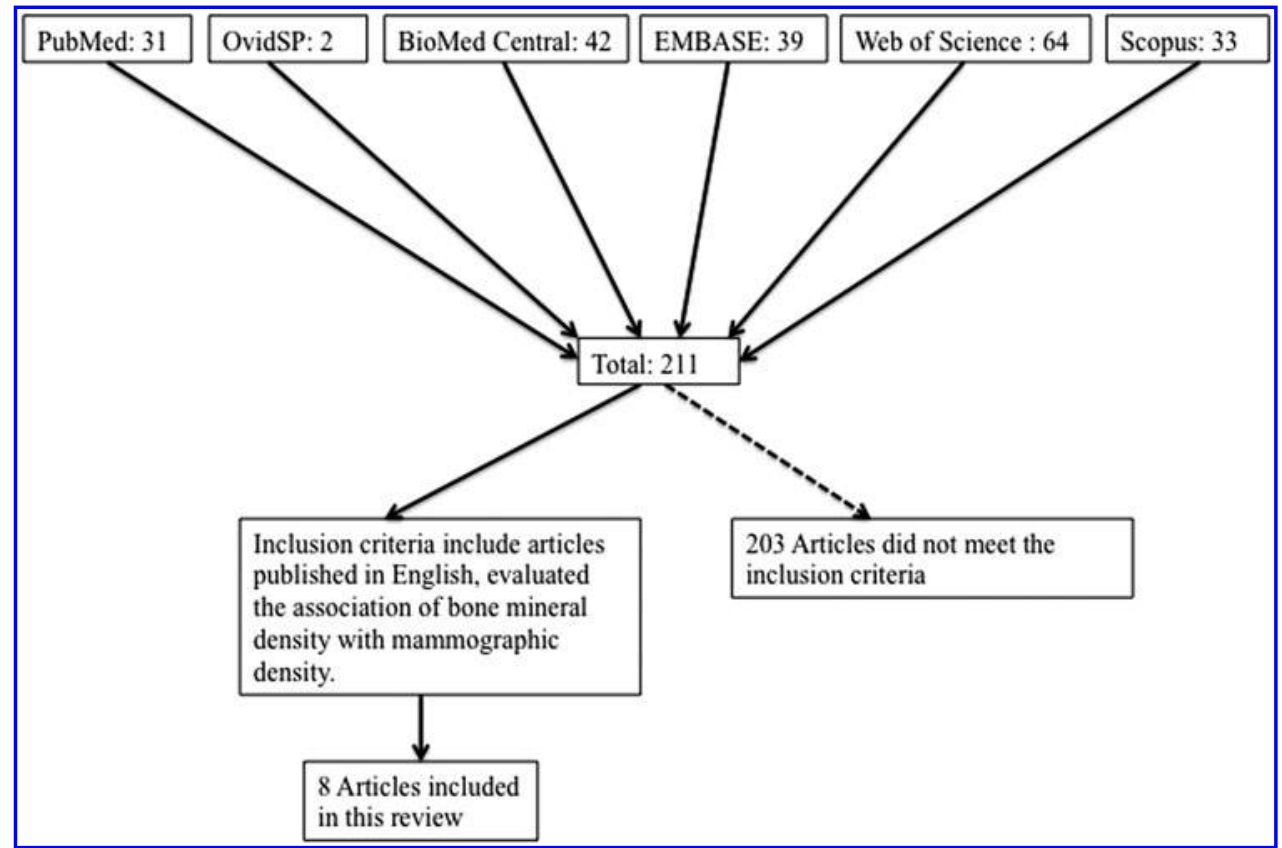


Table 1. Summary Description of Studies on the Association of Bone Mineral Density at the Spine with Percent Mammographic Breast Density

\begin{tabular}{|c|c|c|c|c|c|c|}
\hline $\begin{array}{l}\text { Reference, } \\
\text { country }\end{array}$ & Design & $\begin{array}{c}\text { Age } \\
\text { (years) }\end{array}$ & Study participants & $\begin{array}{c}\text { Results } \\
(\beta \text { coefficient } \\
\text { or correlation })\end{array}$ & $\begin{array}{c}95 \% \\
\text { Confidence } \\
\text { interval/p }\end{array}$ & Confounders \\
\hline $\begin{array}{l}\text { Sung et al., } \\
\text { Korea }\end{array}$ & Cross-sectional & $\geq 30$ & $\begin{array}{l}\text { Overall }(N=730) \\
\text { Premenopausal } \\
\quad(N=462) \\
\text { Postmenopausal } \\
\quad(N=268)\end{array}$ & $\begin{array}{l}\beta=0.27^{\mathrm{a}} \\
\beta=0.21^{\mathrm{a}} \\
\beta=0.42^{\mathrm{a}}\end{array}$ & $\begin{array}{l}-0.01 \text { to } 0.55 \\
-0.0 \text { to } 0.46 \\
-0.27 \text { to } 1.11\end{array}$ & $\begin{array}{l}\text { Age, smoking status, } \\
\text { alcohol } \\
\text { consumption, } \\
\text { physical exercise, } \\
\text { number of live } \\
\text { children, age at } \\
\text { menarche, age at } \\
\text { birth of first child, } \\
\text { duration of breast } \\
\text { feeding, use of oral } \\
\text { contraceptives, } \\
\text { hormone } \\
\text { replacement therapy }\end{array}$ \\
\hline $\begin{array}{l}\text { Gupta et al., } \\
\text { Kuwait }\end{array}$ & Cross-sectional & $25-74$ & $\begin{array}{l}\text { Premenopausal } \\
\quad(N=147) \\
\text { Postmenopausal } \\
(N=96)\end{array}$ & $\begin{array}{l}r=0.158^{b} \\
r=0.021^{b}\end{array}$ & $\begin{array}{l}0.125 \\
0.798\end{array}$ & Ethnicity \\
\hline $\begin{array}{l}\text { Crandall et al., } \\
\text { United States }\end{array}$ & Cross-sectional & $42-52$ & $\begin{array}{l}\text { Early } \\
\quad \text { Perimenopausal } \\
\quad(N=249) \\
\text { Premenopausal } \\
\quad(N=56)\end{array}$ & $\begin{array}{l}\beta=-20.6^{\mathrm{c}} \\
\beta=-29.0^{\mathrm{c}}\end{array}$ & 0.02 & $\begin{array}{l}\text { Age, BMI, ethnicity, } \\
\text { study site, number } \\
\text { of pregnancies, } \\
\text { alcohol } \\
\text { consumption, age at } \\
\text { first childbirth, } \\
\text { smoking status, } \\
\text { physical activity } \\
\text { score }\end{array}$ \\
\hline $\begin{array}{l}\text { Dite et al., } \\
\text { Australia }\end{array}$ & Cross-sectional & N/A & $\begin{array}{l}\text { Overall }(N=268) \\
\text { Premenopausal } \\
(N=\text { N/A }) \\
\text { Postmenopausal/past } \\
\text { or never HT } \\
(N=\text { N/A }) \\
\text { Postmenopausal/ } \\
\text { current or recent } \\
\text { HT users } \\
(N=\text { N/A })\end{array}$ & $\begin{array}{c}\beta=-0.02^{\mathrm{d}} \\
\beta=-0.10^{\mathrm{d}} \\
\beta=0.02^{\mathrm{d}} \\
\beta=0.24^{\mathrm{d}}\end{array}$ & $\begin{array}{l}0.79 \\
0.33 \\
0.85\end{array}$ & $\begin{array}{l}\text { Age, BMI, smoking } \\
\text { status }\end{array}$ \\
\hline $\begin{array}{l}\text { Dite et al., } \\
\text { Australia }\end{array}$ & Longitudinal & $38-71$ & Overall $(N=134)$ & $\mathrm{r}=0.015^{\mathrm{e}}$ & 0.8 & $\begin{array}{l}\text { Age at mammogram, } \\
\text { age at bone scan, } \\
\text { height and weight }\end{array}$ \\
\hline $\begin{array}{l}\text { Crandall et al., } \\
\text { United States }\end{array}$ & Cross-sectional & $45-64$ & $\begin{array}{l}\text { Postmenopausal } \\
\text { women who are } \\
\text { not recent HT } \\
\text { users }(N=417) \\
\text { Postmenopausal } \\
\text { women who are } \\
\text { recent HT users } \\
(N=173)\end{array}$ & $\beta=-0.065^{\mathrm{d}}$ & 0.08 & $\begin{array}{l}\text { Age, BMI, smoking } \\
\text { status }\end{array}$ \\
\hline $\begin{array}{l}\text { Kerlikowske } \\
\text { et al., } \\
\text { United States }\end{array}$ & $\begin{array}{l}\text { Cross-sectional } \\
\text { and nested } \\
\text { case-control } \\
\text { study }\end{array}$ & $\geq 28$ & $\begin{array}{l}\text { Overall }(N=405) \\
\text { Premenopausal } \\
(N=20) \\
\text { Postmenopausal/past } \\
\text { or never HT } \\
(N=142) \\
\text { Postmenopausal/ } \\
\text { current or recent } \\
\text { HT }(N=153)\end{array}$ & $\begin{array}{l}r=0.01^{\mathrm{f}} \\
\mathrm{r}=0.09^{\mathrm{f}} \\
\mathrm{r}=0.06^{\mathrm{f}}\end{array}$ & $\begin{array}{l}0.87 \\
0.71 \\
0.48\end{array}$ & $\begin{array}{l}\text { Age, BMI, ethnicity, } \\
\text { age at first live } \\
\text { birth, HT use }\end{array}$ \\
\hline
\end{tabular}

\footnotetext{
${ }^{a}$ Beta coefficients assessed by a linear mixed model.

'Spearman's correlation coefficient.

${ }^{\mathrm{c}}$ Beta coefficients assessed by a linear regression model. Current/recent hormone users were excluded in this model.

${ }^{\mathrm{d}}$ Beta coefficients assessed by a linear regression model.

${ }^{\mathrm{e}}$ Cross-trait cross-twin correlations.

${ }^{\text {f}}$ Pearson's correlation coefficient.

BMI, body mass index; HT, hormone therapy; N/A, not available.
} 
Table 2. Summary Description of Studies on the Association of Bone Mineral Density at the Hip with Percent Mammographic Breast Density

\begin{tabular}{|c|c|c|c|c|c|c|}
\hline Reference, country & Design & $\begin{array}{c}\text { Age } \\
\text { (years) }\end{array}$ & Study participants & $\begin{array}{c}\text { Results } \\
\text { ( } \beta \text { coefficient or } \\
\text { correlation) }\end{array}$ & $\begin{array}{c}95 \% \\
\text { Confidence } \\
\text { interval/p }\end{array}$ & Confounders \\
\hline $\begin{array}{l}\text { Sung et al., } \\
\text { Korea }\end{array}$ & Cross-sectional & $\geq 30$ & $\begin{array}{l}\text { Overall }(N=730) \\
\text { Premenopausal } \\
\quad(N=462) \\
\text { Postmenopausal } \\
\quad(N=268)\end{array}$ & $\begin{array}{l}\beta=0.75^{\mathrm{a}} \\
\beta=0.59^{\mathrm{a}} \\
\beta=0.45^{\mathrm{a}}\end{array}$ & $\begin{array}{r}0.30 \text { to } 1.21 \\
0.16 \text { to } 1.02 \\
-0.46 \text { to } 1.36\end{array}$ & $\begin{array}{l}\text { Age, smoking status, } \\
\text { alcohol } \\
\text { consumption, } \\
\text { physical exercise, } \\
\text { number of live } \\
\text { children, age at } \\
\text { menarche, age at } \\
\text { birth of first child, } \\
\text { duration of breast } \\
\text { feeding, use of oral } \\
\text { contraceptives, } \\
\text { hormone } \\
\text { replacement } \\
\text { therapy }\end{array}$ \\
\hline $\begin{array}{l}\text { Gupta et al., } \\
\text { Kuwait }\end{array}$ & Cross-sectional & $25-74$ & $\begin{array}{l}\text { Premenopausal } \\
(N=147) \\
\text { Postmenopausal } \\
(N=96)\end{array}$ & $\begin{array}{l}r=0.094^{b} \\
r=0.082^{b}\end{array}$ & $\begin{array}{l}0.385 \\
0.342\end{array}$ & Ethnicity \\
\hline $\begin{array}{l}\text { Crandall et al., } \\
\text { United States }\end{array}$ & Cross-sectional & $42-52$ & $\begin{array}{l}\text { Early } \\
\quad \text { Perimenopausal } \\
\quad(N=301) \\
\text { Premenopausal } \\
\quad(N=80)\end{array}$ & $\begin{array}{r}\beta=-18.8^{\mathrm{c}} \\
\beta=3.9^{\mathrm{c}}\end{array}$ & 0.87 & $\begin{array}{l}\text { Age, BMI, ethnicity, } \\
\text { study site, number } \\
\text { of pregnancies, } \\
\text { alcohol } \\
\text { consumption, age } \\
\text { at first childbirth, } \\
\text { smoking status, } \\
\text { physical activity } \\
\text { score }\end{array}$ \\
\hline $\begin{array}{l}\text { Dite et al., } \\
\text { Australia }\end{array}$ & Cross-sectional & N/A & $\begin{array}{l}\text { Overall }(N=268) \\
\text { Premenopausal } \\
(N=\text { N/A }) \\
\text { Postmenopausal/ } \\
\text { past or never HT } \\
(N=\text { N/A }) \\
\text { Postmenopausal/ } \\
\text { current or recent } \\
\text { HT users } \\
(N=\text { N/A })\end{array}$ & $\begin{array}{c}\beta=-0.09^{\mathrm{d}} \\
\beta=-0.23^{\mathrm{d}} \\
\beta=0.05^{\mathrm{d}}\end{array}$ & $\begin{array}{l}0.41 \\
0.06 \\
0.78\end{array}$ & $\begin{array}{l}\text { Age, BMI, and } \\
\text { smoking status }\end{array}$ \\
\hline $\begin{array}{l}\text { Crandall et al., } \\
\text { United States }\end{array}$ & Cross-sectional & $45-64$ & $\begin{array}{l}\text { Postmenopausal } \\
\text { women who are } \\
\text { not recent HT } \\
\text { users }(N=417) \\
\text { Postmenopausal } \\
\text { women who are } \\
\text { recent HT users } \\
(N=173)\end{array}$ & $\beta=-0.073^{\mathrm{d}}$ & 0.51 & $\begin{array}{l}\text { Age, BMI, smoking } \\
\text { status }\end{array}$ \\
\hline $\begin{array}{l}\text { Kerlikowske } \\
\text { et al., } \\
\text { United States }\end{array}$ & $\begin{array}{l}\text { Cross-sectional } \\
\text { and nested } \\
\text { case-control } \\
\text { study }\end{array}$ & $\geq 28$ & $\begin{array}{l}\text { Overall }(N=424) \\
\text { Premenopausal } \\
(N=22) \\
\text { Postmenopausal/ } \\
\text { past or never HT } \\
(N=149) \\
\text { Postmenopausal/ } \\
\text { current or recent } \\
\text { HT }(N=158)\end{array}$ & $\begin{array}{l}\mathrm{r}=-0.06^{\mathrm{e}} \\
\mathrm{r}=-0.00^{\mathrm{e}} \\
\mathrm{r}=-0.11^{\mathrm{e}}\end{array}$ & $\begin{array}{l}0.22 \\
1.00 \\
0.17\end{array}$ & $\begin{array}{l}\text { Age, BMI, ethnicity, } \\
\text { age at first live } \\
\text { birth, HT use }\end{array}$ \\
\hline
\end{tabular}

\footnotetext{
${ }^{a}$ Beta coefficients assessed by a linear mixed model.

${ }^{\mathrm{b}}$ Spearman's correlation coefficient.

${ }^{c}$ Beta coefficients assessed by a linear regression model. Current/recent hormone users were included in this model.

${ }^{\mathrm{d}}$ Beta coefficients assessed by a linear regression model.

${ }^{\mathrm{e}}$ Pearson's correlation coefficient.
} 
Table 3. Summary Description of Studies on the Association of Bone Mineral Density at Other Sites (Ribs, Arms, Body) with Percent Mammographic Breast Density

\begin{tabular}{|c|c|c|c|c|c|c|}
\hline $\begin{array}{l}\text { Reference, } \\
\text { country }\end{array}$ & Design & $\begin{array}{c}\text { Age } \\
\text { (years) }\end{array}$ & $\begin{array}{c}\text { Study } \\
\text { participants }\end{array}$ & $\begin{array}{c}\text { Results } \\
\text { ( } \beta \text { coefficient } \\
\text { or correlation })\end{array}$ & $\begin{array}{c}95 \% \\
\text { Confidence } \\
\text { interval/p }\end{array}$ & Confounders \\
\hline \multirow{6}{*}{$\begin{array}{l}\text { Moseson } \\
\text { et al., } \\
\text { Mexico }\end{array}$} & \multirow{6}{*}{$\begin{array}{l}\text { Prospective } \\
\text { cohort } \\
\text { study }\end{array}$} & \multirow[t]{6}{*}{ N/A } & \multirow[t]{3}{*}{$\begin{array}{l}\text { Premenopausal } \\
\qquad(N=955)\end{array}$} & $\begin{array}{l}\text { Forearm (BMD: } \\
\quad 0.41-0.45): 0.2^{\mathrm{a}}\end{array}$ & -2.30 to 2.71 & \multirow{6}{*}{$\begin{array}{l}\text { Family history of breast } \\
\text { cancer, age at } \\
\text { menarche, smoking, } \\
\text { alcohol, hormone use, } \\
\text { daily calcium intake, } \\
\text { BMI, number of } \\
\text { pregnancies, age at } \\
\text { first pregnancy, } \\
\text { contraceptive use }\end{array}$} \\
\hline & & & & $\begin{array}{l}\text { Forearm (BMD: } \\
\quad 0.45-0.48):-0.91^{\mathrm{a}}\end{array}$ & -3.42 to 1.61 & \\
\hline & & & & $\begin{array}{l}\text { Forearm (BMD: } \\
0.48+):-0.21^{\mathrm{a}}\end{array}$ & -2.82 to 2.40 & \\
\hline & & & \multirow[t]{3}{*}{$\begin{array}{l}\text { Postmenopausal } \\
\quad(N=552)\end{array}$} & $\begin{array}{l}\text { Forearm (BMD: } \\
0.35-0.40): 0.77^{\mathrm{a}}\end{array}$ & -2.32 to 3.86 & \\
\hline & & & & $\begin{array}{l}\text { Forearm (BMD: } \\
\quad 0.40-0.44): 5.06^{\mathrm{a}}\end{array}$ & 1.87 to 8.26 & \\
\hline & & & & $\begin{array}{l}\text { Forearm (BMD: } \\
0.44+): 4.44^{\mathrm{a}}\end{array}$ & 1.11 to 7.77 & \\
\hline \multirow{12}{*}{$\begin{array}{l}\text { Sung et al., } \\
\text { Korea }\end{array}$} & \multirow{12}{*}{$\begin{array}{l}\text { Cross- } \\
\text { sectional }\end{array}$} & \multirow[t]{12}{*}{$\geq 30$} & Overall $(N=730)$ & Legs: $\beta=0.661$ & 0.16 to 1.15 & \multirow{12}{*}{$\begin{array}{l}\text { Age, smoking status, } \\
\text { alcohol consumption, } \\
\text { physical exercise, } \\
\text { number of live } \\
\text { children, age at } \\
\text { menarche, age at } \\
\text { birth of first child, } \\
\text { duration of breast } \\
\text { feeding, use of oral } \\
\text { contraceptives, } \\
\text { hormone replacement } \\
\text { therapy }\end{array}$} \\
\hline & & & $\begin{array}{l}\text { Premenopausal } \\
\quad(N=462)\end{array}$ & Legs: $\beta=0.59^{\mathrm{a}}$ & 0.05 to 1.14 & \\
\hline & & & $\begin{array}{l}\text { Postmenopausal } \\
\quad(N=268)\end{array}$ & Legs: $\beta=0.39^{\mathrm{a}}$ & -0.51 to 1.28 & \\
\hline & & & Overall $(N=730)$ & Arms: $\beta=0.93^{\mathrm{a}}$ & 0.28 to 1.57 & \\
\hline & & & $\begin{array}{l}\text { Premenopausal } \\
\quad(N=462)\end{array}$ & Arms: $\beta=0.43^{\mathrm{a}}$ & -0.05 to 0.92 & \\
\hline & & & $\begin{array}{l}\text { Postmenopausal } \\
\quad(N=268)\end{array}$ & Arms: $\beta=1.87^{\mathrm{a}}$ & -0.09 to 3.82 & \\
\hline & & & Overall $(N=730)$ & Ribs: $\beta=1.11^{\mathrm{a}}$ & 0.29 to 1.92 & \\
\hline & & & $\begin{array}{l}\text { Premenopausal } \\
\quad(N=462)\end{array}$ & Ribs: $\beta=0.59^{\mathrm{a}}$ & -0.07 to 1.24 & \\
\hline & & & $\begin{array}{l}\text { Postmenopausal } \\
\quad(N=268)\end{array}$ & Ribs: $\beta=0.91^{\mathrm{a}}$ & -1.53 to 3.36 & \\
\hline & & & Overall $(N=730)$ & $\begin{array}{l}\text { Whole body: } \\
\qquad \beta=0.381\end{array}$ & -0.14 to 0.90 & \\
\hline & & & $\begin{array}{l}\text { Premenopausal } \\
\quad(N=462)\end{array}$ & $\begin{array}{l}\text { Whole body: } \\
\qquad \beta=0.46^{\mathrm{a}}\end{array}$ & -0.10 to 1.03 & \\
\hline & & & $\begin{array}{l}\text { Postmenopausal } \\
\quad(N=268)\end{array}$ & $\begin{array}{l}\text { Whole body: } \\
\qquad \beta=0.005^{\mathrm{a}}\end{array}$ & -0.89 to 0.90 & \\
\hline \multirow{2}{*}{$\begin{array}{l}\text { Dite et al., } \\
\text { Australia }\end{array}$} & \multirow[t]{2}{*}{ Longitudinal } & \multirow[t]{2}{*}{$38-71$} & Overall $(N=134)$ & Arms: $r=0.0702$ & 0.3 & \multirow{2}{*}{$\begin{array}{l}\text { Age at mammogram, } \\
\text { age at bone scan, } \\
\text { height and weight }\end{array}$} \\
\hline & & & Overall $(N=134)$ & Neck: $r=-0.031^{\mathrm{b}}$ & 0.6 & \\
\hline
\end{tabular}

${ }^{\mathrm{a}}$ Beta coefficients assessed by a linear mixed model.

${ }^{\mathrm{b}}$ Cross-trait cross-twin correlations.

BMD, bone mineral density.

among postmenopausal women, but not among premenopausal women (Table 3).

\section{Discussion}

In this review, we identified eight studies published up till April 2016 that evaluated the association of BMD with percent mammographic density. There is little evidence to support an association between BMD and percent mammographic density. However, there appears to be a weak association between BMD at the spine and hip and percent mammographic density, which may be modified by menopausal status. While BMD at the spine was weakly positively associated with percent mammographic density among postmenopausal women who were not hormone users, BMD at the spine and hip was weakly negatively associated with mammographic density among perimeno- pausal women. On the other hand, BMD at the hip and legs was weakly positively associated with percent mammographic density among premenopausal women. Since reproductive hormone changes across a menstrual cycle as well as across the menopausal transition, ${ }^{35}$ it is possible that menopausal status, which affects reproductive hormone levels, modifies the effect of BMD on percent mammographic density.

\section{Hormonal status, BMD, and mammographic density}

A few of the studies observed a small, but statistically significant association of BMD on percent mammographic density after stratifying by menopausal status. This suggests that changes in reproductive hormone levels, particularly estrogen, over the course of the reproductive life cycle might moderate the association of BMD and percent mammographic density. 
Estrogen levels in women decrease with aging. ${ }^{36,37}$ The loss of ovarian estrogen during menopause is associated with a decline in BMD. ${ }^{17,37}$ Postmenopausal women who use menopausal estrogen therapy for more than 7 years have higher BMD than those who had not taken estrogen therapy. ${ }^{38}$ Furthermore, changes in reproductive hormones affect breast density as well, ${ }^{7}$ although this appears mainly due to progesterone. ${ }^{11}$ This increase in percent mammographic density was greater among postmenopausal women with the use of estrogen/progestin combination therapy, but not with the use of estrogen only. ${ }^{39}$ Therefore, studies evaluating the associations of BMD with mammographic density need to consider hormonal status, with respect to menopausal status and use of menopausal hormonal therapy. Of the seven studies in this review, only four stratified their analyses by both menopausal status and use of menopausal hormonal therapy. ${ }^{27,29-31}$

Furthermore, because combined menopausal hormone therapy (estrogen plus progestin) impacts breast density rather than estrogen alone, it is important for future studies to specify what type of menopausal hormone therapy study participants used.

\section{$B M I, B M D$, and mammographic density}

There is a complex relationship between adiposity, breast cancer and breast cancer risk factors. Elevated BMI during the premenopausal years has a protective effect on premenopausal breast cancer, but during the postmenopausal years, elevated BMI is associated with increased breast cancer risk. ${ }^{40,41}$ In addition, increased BMI is positively associated with BMD, but inversely associated with mammographic density. ${ }^{42-44}$ For postmenopausal women, as BMI increases, estrogen levels increase due to the conversion of androgens to estrogen in adipose tissue. ${ }^{45}$ In addition, a recently published study reported no association overall, but a positive association between BMD and percent mammographic density among obese women, and an inverse association among leaner women. ${ }^{34}$ This suggests a complex association. Nevertheless, studies evaluating the association of BMD with percent mammographic density have not stratified their analyses by BMI, except for one conducted among Kuwaiti women. ${ }^{32}$ It should be noted that one study not included in this review, because it reported breast density using the BI-RADS classification rather than percent mammographic density, reported a small, but significant negative association between BMD at hip and breast density among postmenopausal women with normal weight and no relationship in overweight or obese women. ${ }^{46}$ Scattered fibroglandular densities and heterogeneously dense categories have wide percent mammographic density ranges and have large overlaps of percentage density in qualitative BI-RADS categories $2-4$, and this may introduce limitations to their study. ${ }^{47,48}$

The limitations of this review need to be taken into consideration when interpreting the results. Because of the heterogeneity of studies and limited data on each BMD site, we performed a systematic review, rather than a meta-analysis, hence no additional statistical analyses were conducted.

In conclusion, there is no evidence to support an association between BMD and percent mammographic density. Any potential association is weak at best, which argues against the need for further studies.

\section{Acknowledgments}

A.T.T. is supported by the Siteman Cancer Center, BarnesJewish Hospital Foundation, Washington University School of Medicine Faculty Diversity Award and Susan G Komen Career Catalyst Award. The funding sources have role of study sponsors, if any, in the study design, collection, analysis, and interpretation of data; in the writing of the article; and in the decision to submit the article for publication.

\section{Author Disclosure Statement}

C.A. is a consultant for Hologic, Inc. All other authors have no competing financial interests.

\section{References}

1. Oza AM, Boyd NF. Mammographic parenchymal patterns: A marker of breast cancer risk. Epidemiol Rev 1993;15:196-208.

2. Keller BM, McCarthy AM, Chen J, et al. Associations between breast density and a panel of single nucleotide polymorphisms linked to breast cancer risk: A cohort study with digital mammography. BMC Cancer 2015;15:143.

3. Boyd NF, Guo H, Martin LJ, et al. Mammographic density and the risk and detection of breast cancer. $\mathrm{N}$ Engl J Med 2007;356:227-236.

4. Bertrand KA, Scott CG, Tamimi RM, et al. Dense and nondense mammographic area and risk of breast cancer by age and tumor characteristics. Cancer Epidemiol Biomarkers Prev 2015;24:798-809.

5. Buist DS, LaCroix AZ, Barlow WE, White E, Weiss NS. Bone mineral density and breast cancer risk in postmenopausal women. J Clin Epidemiol 2001;54:417-422.

6. Kim BK, Choi YH, Song YM, et al. Bone mineral density and the risk of breast cancer: A case-control study of Korean women. Ann Epidemiol 2014;24:222-227.

7. Greendale GA, Reboussin BA, Sie A, et al. Effects of estrogen and estrogen-progestin on mammographic parenchymal density. Postmenopausal Estrogen/Progestin Interventions (PEPI) Investigators. Ann Intern Med 1999;130:262-269.

8. Dumas I, Diorio C. Polymorphisms in genes involved in the estrogen pathway and mammographic density. BMC Cancer 2010;10:636.

9. Tamimi RM, Hankinson SE, Colditz GA, Byrne C. Endogenous sex hormone levels and mammographic density among postmenopausal women. Cancer Epidemiol Biomarkers Prev 2005;14:2641-2647.

10. Aiello EJ, Tworoger SS, Yasui Y, et al. Associations among circulating sex hormones, insulin-like growth factor, lipids, and mammographic density in postmenopausal women. Cancer Epidemiol Biomarkers Prev 2005;14:1411-1417.

11. McTiernan A, Martin CF, Peck JD, et al. Estrogen-plusprogestin use and mammographic density in postmenopausal women: Women's Health Initiative randomized trial. J Natl Cancer Inst 2005;97:1366-1376.

12. Clemons M, Goss P. Estrogens and the risk of breast cancer. N Engl J Med 2001;344:276-285.

13. Murphy S, Khaw KT, Sneyd MJ, Compston JE. Endogenous sex hormones and bone mineral density among community-based postmenopausal women. Postgrad Med J 1992;68:908-913.

14. Corina M, Vulpoi C, Branisteanu D. Relationship between bone mineral density, weight, and estrogen levels in pre and postmenopausal women. Rev Med Chir Soc Med Nat Iasi 2012;116:946-950. 
15. Nguyen TV, Jones G, Sambrook PN, White CP, Kelly PJ, Eisman JA. Effects of estrogen exposure and reproductive factors on bone mineral density and osteoporotic fractures. J Clin Endocrinol Metab 1995;80:2709-2714.

16. Weitzmann MN, Pacifici R. Estrogen deficiency and bone loss: An inflammatory tale. J Clin Invest 2006;116:1186-1194.

17. Vaananen HK, Harkonen PL. Estrogen and bone metabolism. Maturitas 1996;23 Suppl:S65-S69.

18. Imai Y, Youn MY, Kondoh S, et al. Estrogens maintain bone mass by regulating expression of genes controlling function and life span in mature osteoclasts. Ann N Y Acad Sci 2009;1173 Suppl 1:E31-E39.

19. Kameda T, Mano H, Yuasa T, et al. Estrogen inhibits bone resorption by directly inducing apoptosis of the boneresorbing osteoclasts. J Exp Med 1997;186:489-495.

20. Eliassen AH, Colditz GA, Rosner B, Willett WC, Hankinson SE. Adult weight change and risk of postmenopausal breast cancer. JAMA 2006;296:193-201.

21. Hunter DJ, Willett WC. Diet, body size, and breast cancer. Epidemiol Rev 1993;15:110-132.

22. Albala C, Yanez M, Devoto E, Sostin C, Zeballos L, Santos JL. Obesity as a protective factor for postmenopausal osteoporosis. Int J Obes Relat Metab Disord 1996;20:1027-1032.

23. Mendez JP, Rojano-Mejia D, Pedraza J, et al. Bone mineral density in postmenopausal Mexican-Mestizo women with normal body mass index, overweight, or obesity. Menopause 2013;20:568-572.

24. Dorgan JF, Klifa C, Shepherd JA, et al. Height, adiposity and body fat distribution and breast density in young women. Breast Cancer Res 2012;14:R107.

25. Schetter SE, Hartman TJ, Liao J, et al. Differential impact of body mass index on absolute and percent breast density: Implications regarding their use as breast cancer risk biomarkers. Breast Cancer Res Treat 2014;146:355-363.

26. Kanis JA. Diagnosis of osteoporosis and assessment of fracture risk. Lancet 2002;359:1929-1936.

27. Dite GS, Wark JD, Giles GG, English DR, McCredie MR, Hopper JL. Is there a positive association between mammographic density and bone mineral density? Breast Cancer Res 2006;8:401.

28. Dite GS, Wark JD, Giles GG, English DR, McCredie MR, Hopper JL. Is there overlap between the genetic determinants of mammographic density and bone mineral density? Cancer Epidemiol Biomarkers Prev 2005;14:2266-2268.

29. Crandall C, Palla S, Reboussin BA, Ursin G, Greendale GA. Positive association between mammographic breast density and bone mineral density in the Postmenopausal Estrogen/Progestin Interventions Study. Breast Cancer Res 2005;7:R922-R928.

30. Crandall CJ, Zheng Y, Karlamangla A, et al. The association between mammographic breast density and bone mineral density in the study of women's health across the nation. Ann Epidemiol 2007;17:575-583.

31. Kerlikowske K, Shepherd J, Creasman J, Tice JA, Ziv E, Cummings SR. Are breast density and bone mineral density independent risk factors for breast cancer? J Natl Cancer Inst 2005;97:368-374.

32. Gupta R, Alhajri FA, Mohammed AM, Chavan VN, Gupta P. Relationship between mammography breast density and bone mineral density. J Clin Densitom 2008;11:431-436.

33. Sung J, Song YM, Stone J, Lee K. The relationship between bone mineral density and mammographic density in Korean women: The Healthy Twin study. Breast Cancer Res Treat 2011;129:583-591.
34. Moseson H, Rice MS, López-Ridaura R, et al. Bone mineral density and mammographic density in Mexican women. Cancer Causes Control 2016;27:39-46.

35. Santoro N, Randolph JF Jr. Reproductive hormones and the menopause transition. Obstet Gynecol Clin North Am 2011;38:455-466.

36. Khosla S, Riggs BL, Robb RA, et al. Relationship of volumetric bone density and structural parameters at different skeletal sites to sex steroid levels in women. J Clin Endocrinol Metab 2005;90:5096-5103.

37. Cauley JA. Estrogen and bone health in men and women. Steroids 2015;99:11-15.

38. Felson DT, Zhang Y, Hannan MT, Kiel DP, Wilson PW, Anderson JJ. The effect of postmenopausal estrogen therapy on bone density in elderly women. $\mathrm{N}$ Engl J Med 1993;329:1141-1146.

39. Greendale GA, Reboussin BA, Slone S, Wasilauskas C, Pike $\mathrm{MC}$, Ursin G. Postmenopausal hormone therapy and change in mammographic density. J Natl Cancer Inst 2003;95:30-37.

40. Montazeri A, Sadighi J, Farzadi F, et al. Weight, height, body mass index and risk of breast cancer in postmenopausal women: A case-control study. BMC Cancer 2008;8:278.

41. Loi S, Milne RL, Friedlander ML, et al. Obesity and outcomes in premenopausal and postmenopausal breast cancer. Cancer Epidemiol Biomarkers Prev 2005;14:1686-1691.

42. Lloyd JT, Alley DE, Hawkes WG, Hochberg MC, Waldstein SR, Orwig DL. Body mass index is positively associated with bone mineral density in US older adults. Arch Osteoporos 2014;9:175.

43. Zhu K, Hunter M, James A, Lim EM, Walsh JP. Associations between body mass index, lean and fat body mass and bone mineral density in middle-aged Australians: The Busselton Healthy Ageing Study. Bone 2015;74:146-152.

44. Brisson J, Morrison AS, Kopans DB, et al. Height and weight, mammographic features of breast tissue, and breast cancer risk. Am J Epidemiol 1984;119:371-381.

45. Calle EE, Kaaks R. Overweight, obesity and cancer: Epidemiological evidence and proposed mechanisms. Nat Rev Cancer 2004;4:579-591.

46. Buist DS, Anderson ML, Taplin SH, LaCroix AZ. The relationship between breast density and bone mineral density in postmenopausal women. Cancer 2004;101:1968-1976.

47. Nicholson BT, LoRusso AP, Smolkin M, Bovbjerg VE, Petroni GR, Harvey JA. Accuracy of assigned BI-RADS breast density category definitions. Acad Radiol 2006;13: 1143-1149.

48. Martin KE, Helvie MA, Zhou C, et al. Mammographic density measured with quantitative computer-aided method: Comparison with radiologists' estimates and BI-RADS categories. Radiology 2006;240:656-665.

Address correspondence to: Adetunji T. Toriola, MD, MPH, PhD Division of Public Health Sciences Department of Surgery Siteman Cancer Center Washington University School of Medicine 660 South Euclid Avenue

Campus Box 8100 St. Louis, MO 63110

E-mail: a.toriola@wustl.edu 